\title{
Designing and Implementing a Framework for Process-Oriented Logistics-Costs Measurement in an Automotive-Supplier Group
}

\author{
Gregor von Cieminski, Michael Karrer, and Malte Zur \\ ZF Friedrichshafen AG, Ehlersstrasse 50, D-88038 Friedrichshafen \\ \{Gregor.Cieminski, Michael.Karrer, Malte.Zur\}@ZF.com
}

\begin{abstract}
Measurements of logistics costs are necessary for manufacturing companies to be able to evaluate the cost effects and trade-offs of logistics management decisions. This case study highlights the challenges $\mathrm{ZF}$ Friedrichshafen AG faced during the implementation of a process-oriented logistics-costs measurement framework. The discussion of possible solutions to these challenges leads to recommendations for research as well as industrial practice. Specifically, a greater degree of co-operation between logisticians and cost accountants seems necessary in both academia and industry in order to develop more standardized logistics-costs measurement methods.
\end{abstract}

Keywords: performance measurement, logistics costs, case study.

\section{Introduction}

The paper reports on an industrial case study of the design and implementation of a process-oriented logistics-costs measurement framework at ZF Friedrichshafen AG, a German automotive supplier. ZF's objective was to design and implement this framework so that it would be both, founded in theory and workable in industrial practice. The main purpose of the paper is to highlight the challenges the company faced in this process, to identify gaps in the theory and to provide companies engaged in comparable projects with practical recommendations. The paper presents basic background information about the company and the motivation behind the cost-measurement approach covered in the case study. The conceptual foundations of the approach are explained. The paper focuses on the challenges $\mathrm{ZF}$ faced during the implementation process and the theoretical and practical implications arising from them. The discussion of these culminates in the paper's conclusions.

\section{Company and Project Background}

ZF Friedrichshafen AG is a leading automotive supplier for driveline and chassis technology. In 2008, ZF's total sales amounted to $€ 12.5$ bn and the company employed around 62,000 staff in its 125 worldwide production locations and over 40 sales and service centers. ZF encompasses 6 divisions and 5 business units 
manufacturing a range of driveline and chassis components - such as transmissions, axle systems, suspension systems and steering systems - for the automotive, marine railway and aviation industries.

The company approaches its target markets as a technology leader, emphasizing the innovativeness, superior functionality and quality of its products. Increasingly, the ZF Group aims to provide its customers superior delivery performance as an integral part of its competitive strategy. The drive for improving the group's logistic performance is reflected in the 2008/2009 "Year of Logistics". This group-wide development program encompasses dedicated improvement initiatives for the ZF logistics function. In order to underline the strategic importance of the logistics function for ZF's business, it was decided to define four logistics key performance indicators (KPI's) to be reported to the executive board of the group, namely the delivery reliability of the group and of its suppliers, the inventory turnover rate and the logistics costs. Whereas for the first three KPI's standardized measurement methods and systems had already been put in place, these were missing for the logistics costs indicator [1]. Therefore $\mathrm{ZF}$ initiated a group-wide project to design and implement a logistics-costs measurement framework.

\section{Framework Design and Implementation Approach}

The project "Transparency of Logistics Costs" consists of two major phases: Firstly the design of the logistics-costs measurement framework and secondly the implementation of the framework in the various locations of the ZF Group. The work content of both phases will be briefly explained.

A team of experts from the logistics and controlling (management accounting) functions was formed in order to develop a logistics-costs measurement framework and prepare its implementation. The remit of the team was to design the framework in such a way that it would be founded in theory and workable in industrial practice. For this reason, the team decided to design a framework with a comprehensive logisticsprocess orientation, but also to consciously discard activity-based costing principles. The implementation of frameworks built on the latter costing method was deemed to require an excessive amount of effort.

Thus, the logistics-costs measurement framework is founded upon four main elements:

1. The ZF logistics reference process model which is based on the Supply-chain Operations Reference (SCOR) model [2]. The first hierarchical level of this model consists of the four SCOR processes Plan, Source, Make, Deliver. At the second level these processes are disaggregated into 14 so-called "sub processes" as shown in Fig. 1.a. The logistics costs are measured and reported with respect to these sub processes.

2. The ZF standardized logistics-costs components, a set of 15 aggregated types of logistics costs shown in Fig. 1.b, which complies with logistics-costs definitions provided in the relevant literature (e.g. [3]). The main function of each of the cost components is to aggregate a number of logistics costs accounts into standardized cost categories. The need to aggregate the accounts resulted from their nonstandardized definition in the different locations of the ZF Group (see 3.). The 
logistics costs are measured, but not officially reported, with respect to the logistics-costs components.

3. The logistics-costs accounts defined in the charts of accounts of the various divisions (locations) of the ZF Group. As stated above, these are specific to the divisions, or indeed locations, of the group.

4. "Logistically-relevant" cost centers, i.e. all cost centers that carry out logistics activities. This includes dedicated logistics cost centers as well as cost centers that carry out a limited number of logistics tasks but are not logistics cost centers as such, e.g. sourcing, production or sales cost centers.
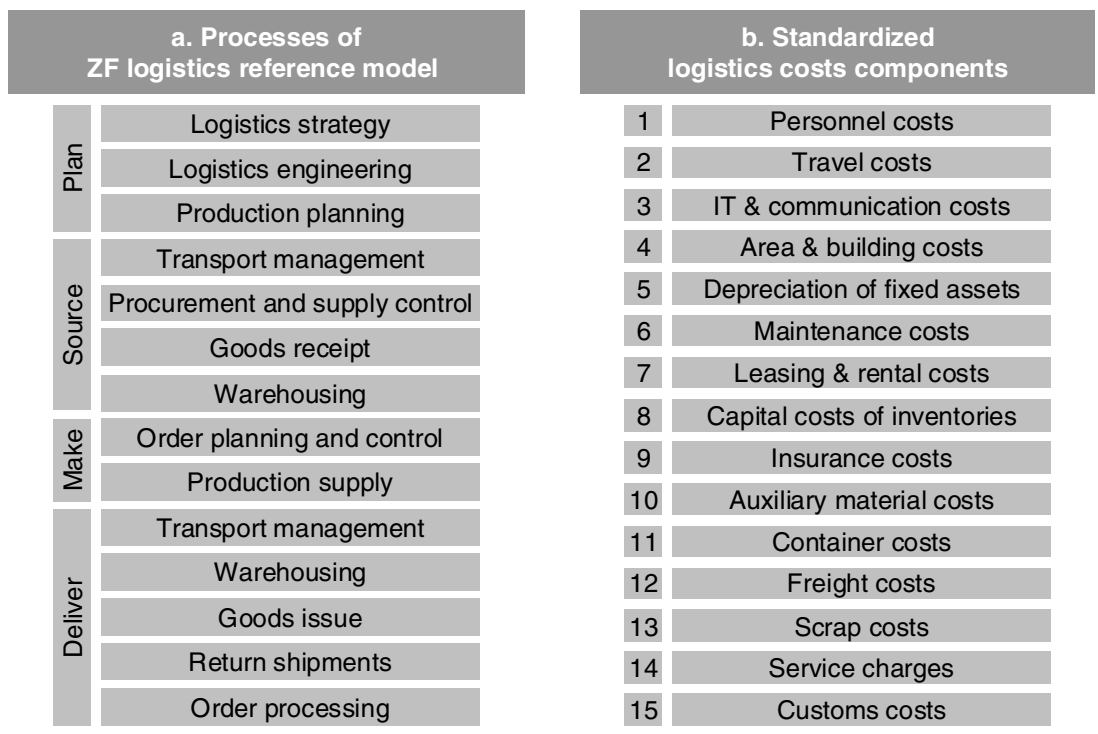

Fig. 1. Logistics processes and logistics-cost components as basic elements of cost-measurement framework

Within the logistics cost-measurement framework, the actual cost data calculated for the cost accounts and cost centers at the locations of the ZF Group have to be allocated to both the logistics processes and the logistics-costs components in order to be able to take the cost measurements required. For the purpose of taking initial manual measurement at the locations according to the proposed framework, a spreadsheet template was designed. This template is used as a tool for logistics-costs measurement workshops that constitute the first step of the dissemination and implementation process of the measurement framework.

The workshop procedure is shown in Fig. 2. It consists of five steps, each of which serves a specific purpose in the context of the measurement methodology. Steps 1 and 2 collect basic information from a ZF location's cost centre structure and chart of accounts. As part of step 2 the first cost allocation, namely to assign the cost accounts to the logistics cost components, is also carried out. In step 3, the costs booked into the respective accounts are accumulated in terms of the logistics-costs components so 
that sums for the components may be calculated for each logistically-relevant cost centre. In step 4 , the sums for the logistics-costs components are further allocated to the logistics processes for these cost centers. Finally, step 5 aggregates the logistics costs of the relevant cost centers and process-based cost figures are calculated for the entire ZF location or reporting unit. Also, the logistics costs are expressed as a percentage of sales, the definition of the top-level logistics costs KPI.

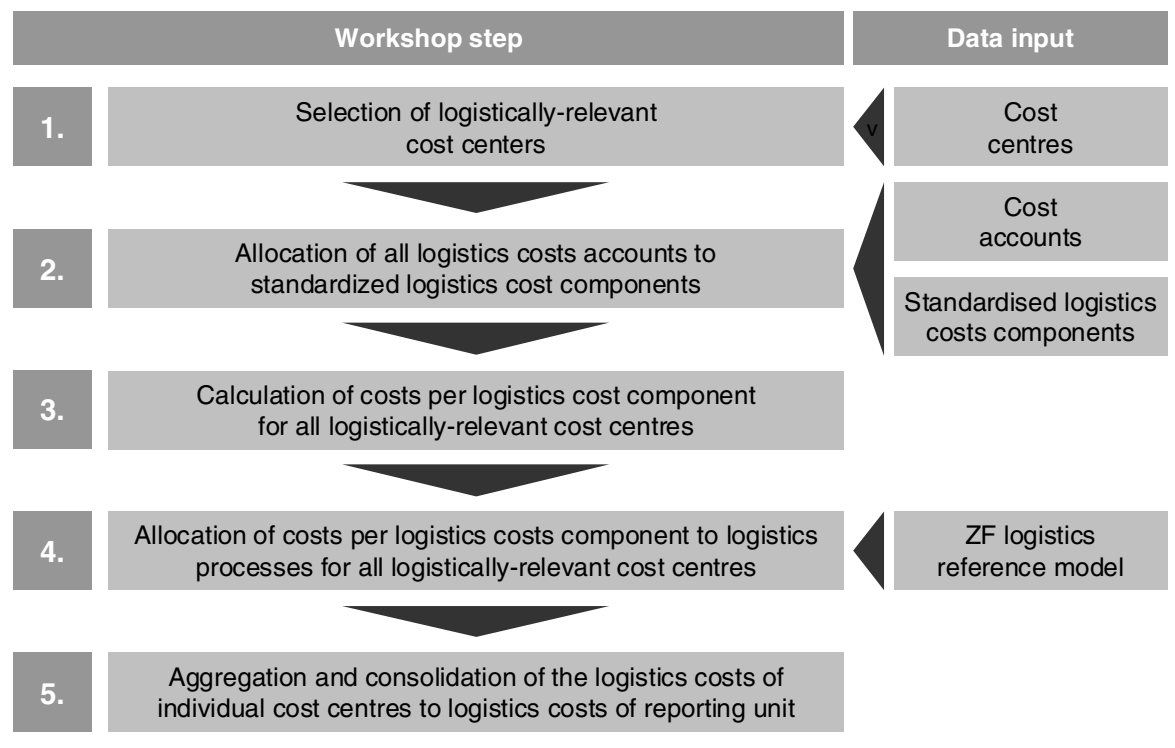

Fig. 2. Workshop procedure for allocating logistics costs

To complete the framework implementation, the results of the cost measurement workshops are transferred from the workshop spreadsheet to the so-called "logistics costs monitor", an evaluation tool that has been integrated into ZF's ERP system. Using this, the logistics-costs reports are to be produced automatically on a monthly basis.

\section{Critical Evaluation of Cost Measurement Framework}

The immediate benefit of the implementation of the logistics-costs measurement framework is to fulfill top management's requirement of a logistics costs KPI. ZF's aim is to measure and report around $80 \%$ of the group's logistics costs using the corporate framework by the end of 2009. This requires the implementation of the framework in around 30 of the group's major locations from different divisions and business units. Despite its "practical inaccuracies", the use of the standardized logistics-cost measurement approach enables ZF to collect cost figures for the logistics function that are comparable across the company's divisions for the first time. The cost measurements assist logistics managers in predicting the cost effects of planning 
and control actions as well as of logistics-process improvements. Also, the cost tradeoffs across the boundaries of the logistics processes that result from pursuing a certain course of action become more readily quantifiable. In order to further enhance the utility of the logistics-costs measurement framework, the company intends to use it as a basis for a formal logistics-costs management process. By combining the measurement framework with an inter-dependency framework of the cost effects of logistics management actions, the logistics staff are provided with a clear indication of the economic viability of their management decisions.

In the design and implementation of the logistics-costs measurement framework, the authors were able to identify gaps in the theory of logistics-costs measurement. Also, the strengths and weaknesses of the $\mathrm{ZF}$ approach can be developed into general practical recommendations.

\subsection{Implications for Academic Research}

The authors' manifest objective was to develop and implement the ZF logistics-costs measurement framework in accordance with current performance measurement theory. Thus, they were able to identify apparent conceptual gaps:

1. The aggregation of collections of the logistics-costs metrics available in the literature references, such as those provided by the SCOR model [2], requires companies to develop their own metrics at higher levels of detail. In this context, more research is required for the "real" standardization of single metrics or frameworks of metrics as pointed out by von Cieminski et al. [4]. Also, judging from the cost accounting practice found in international locations of the ZF Group, there is a lack of standardization of the charts of accounts of manufacturing companies. Indeed, in a - somewhat limited - literature review, the authors were not able to identify a reference to standard charts of accounts specifically defined for the manufacturing industries.

2. Echoing findings by Bourne et al [5], only few references were identified that suggest detailed procedures for the implementation of performance measurement frameworks. Evidently, there is a need for action research projects that aim to develop such procedures which are applicable for different types of performance metrics and different types of companies.

3. As yet, there seems to be insufficient knowledge transfer between the research areas of logistics and supply chain management on the one hand and cost accounting on the other hand. The underlying principles of both areas are not readily compatible. The cost information yielded by standard cost-accounting principles, i.e. allocating costs to cost centers and cost accounts does not easily facilitate a "clear and fair" allocation of logistics costs. Conversely, process orientation is one of the overriding principles of current logistics thinking. Aspects of logistic performance as well as logistics costs are therefore normally considered with respect to logistics processes. This logistic performance measurement principle is set in contrast to the cost-centre orientation found in cost-accounting theory and practice. Thus, if the objective of a company's logistics-costs measurement system is to provide a process orientation, complicated cross-allocations between the cost-centre structure already in place and the structure of the logistics processes are required. Therefore, there is a need to define the structure of logistics cost centers in consideration of 
the process view of the logistics function. Despite the abundance of publications on activity-based costing in relation to logistics, this is an aspect that is not widely covered in the literature.

\subsection{Implications for Industrial Practice}

On the basis of the experience of the framework implementation process, a number of general practical recommendations can be made. These relate to the design of costmeasurement frameworks as well as the implementation and use of such frameworks.

1. The ZF logistics reference model provides a relatively aggregated definition of logistics processes. Models of this type offer the advantage of being valid even for those locations of a company which are not fully structured in accordance with standardized, and detailed, process models. At the same time, process models serve to arrive at the common understanding of logistics activities that is required in order to establish standardized logistics-cost measurements across industrial groups.

2. For reasons of practicality, the logistics-costs measurement framework is implemented as a descriptive rather than prescriptive performance measurement tool. Standard definitions of the elements of the logistics-costs reports, the logistics processes and the logistics-cost components, exist. However, there is no binding definition of the mode of allocation of the cost accounts and cost centers to these standard elements that the ZF locations are obliged to use. Therefore, in the implementation process, the emphasis was placed on collecting the logistics costs as completely as possible and not necessarily as "correctly" as possible. Thus, although the approach fulfilled its purpose in terms of practical applicability, its limitations in terms of accuracy have to be consciously considered.

3. The implementation process in the different ZF locations highlighted the lack of standardization of the group's charts of accounts as well as the cost centre structures. Obviously, this has an adverse effect on the comparability of the logisticscosts reports across the ZF divisions and locations. As a consequence, two recommendations can be made:

- Fundamentally, there is a need for standardizing the chart of accounts of the ZF Group so as to increase the comparability of the logistics-costs reports. Realistically, the standardization process requires organizational changes of such a magnitude that it would have been unrealistic in terms of effort and time-scale to combine it with the implementation of the logistics-costs measurement framework. From the particular perspective of the project, it would be of special importance to consider the requirements of the logistics function in the definition of the standard accounts. Care should be taken, to define accounts that inherently incorporate a meaningful orientation

- The experience gained during the implementation of the framework shows that a cost-centre structure defined with regard to the logistics processes not only greatly simplifies the allocation of logistics costs. It also leads to an unambiguous allocation of costs according to the process orientation. Therefore, as much as is practically possible cost centers should be defined in such a way that they are responsible for a single logistics process

4. One of the main advantages of a process-oriented framework for logistics-costs measurement is its facility to reveal cost trade-offs across process boundaries. In 
order to effectively manage logistics costs it was found to be insufficient to merely provide a transparency of the logistics costs. Additionally, staff responsible for logistics costs also have to be provided with structured action frameworks so that the right decisions may be taken on the basis of the logistics-costs measurements. For logistics and other areas of business management characterized by complex decisions contexts, this aspect can be generalized: Providing operational practitioners with performance measurements does not fully help them to take appropriate decisions. The effects of the various decision alternatives on the aspects of performance considered have to be made obvious as well.

\section{Conclusions}

The evaluation of a case study of the implementation of a logistics-costs measurement framework in an automotive supplier group in this paper leads to the identification of valuable future research activities in the context of logistics-cost measurement and management as well as to practical recommendations for these areas of logistics management. A general conclusion is that action research projects of performance measurement system implementations would be beneficial for improving the understanding the requirements of the implementation process itself and of the particular requirements that logistics practitioners have of performance measurement systems to help them in their day-to-day management tasks. An aspect the paper does not consider is the definition of appropriate change management approaches for the introduction of new performance measurement systems. In the opinion of the authors, this also represents an area that has to be further addressed in research and practice.

\section{References}

1. Karrer, M., Zur, M.: Logistikkostentransparenz zur Erhöhung der Supply ChainPerformance - Erfahrungen der ZF Friedrichshafen AG. In: Proceedings of Supply Chain Controlling and Performance Management Conference, Berlin (2009)

2. Supply Chain Council: Supply-Chain Operations Reference model (SCOR) Version 9.0, http: / / www . supply-chain.org

3. Lin, B., Collins, J., Su, R.K.: Supply chain costing: an activity-based perspective. Int. J. Physical Dist. \& Logistics Mgmt. 31, 702-713 (2001)

4. Von Cieminski, G., Nyhuis, P.: Developing a Coherent Logistic Performance Measurement System for Manufacturing Companies. In: Smeds, R., et al. (eds.) Innovations in Networks: Proceedings of the IFIP WG5.7 APMS 2008 Conference. Helsinki University of Technology, Espoo (2008)

5. Bourne, M., Mills, J., Wilcox, M., Neely, A., Platts, K.: Designing, implementing and updating performance measurement systems. Int. J. Oper. Prod. Mgmt. 20, 754-771 (2000) 